Palavras chave:

Nutrição mineral

Espécies florestais nativas

Produção de mudas

Histórico:

Recebido 05/05/20 I I

Aceito 13/12/2013

Keywords:

Mineral nutrition

Native forest species

Production of seedlings

Correspondência: elzimar.goncalves@ufes.br

DOI: | $0.1590 / 0104776020 \mid 42003$ | 220
Elzimar de Oliveira Gonçalves', Haroldo Nogueira de Paiva², Julio Cesar Lima Neves², Valéria Hollunder Klippel ${ }^{2}$, Marcos Vinicius Winckler Caldeira ${ }^{3}$

\section{CRESCIMENTO DE JACARANDÁ-DA-BAHIA (Dalbergia nigra((Vell.) Fr. All. ex Benth)) SOB DIFERENTES DOSES DE NPK}

RESUMO: O uso de mudas bem nutridas e de qualidade, é um dos fatores que contribui para o sucesso da implantação de plantios florestais. Neste estudo, objetivou-se verificar por meio de características morfológicas, os efeitos dos nutrientes nitrogênio, fósforo e potássio no crescimento de mudas do jacarandá-da-bahia (Dalbergia nigra ((Vell.) Fr. All. para estabelecer a dose ideal de cada nutriente a ser adicionada em diferentes substratos, visando a garantir a produção de mudas com melhor qualidade. Para tanto, as mudas foram produzidas, em três tipos de solos: Argissolo Vermelho-amarelo mestrófico (PVAm), Latossolo Vermelho-amarelo álico (LVAa) e Latossolo Vermelho-amarelo distrófico (LVAd). O experimento foi conduzido em casa de vegetação no período de fevereiro de 2006 a junho de 2006. Foram delimitados II tratamentos, segundo uma matriz baconiana, onde se variaram os nutrientes ( $N, P$ e $K$ ) em três doses diferentes e dois tratamentos adicionais (zero e referência). Adotou-se o delineamento inteiramente casualizado com quatro repetições. As mudas foram plantadas em vasos com capacidade de $2,1 \mathrm{dm}^{3}$, e após 120 dias da semeadura foram coletados as dados. Após análise dos dados, concluiu-se que para a produção de mudas de jacarandá-da-bahia recomenda-se a adição doses iguais ou superiores a $600 \mathrm{mg} \cdot \mathrm{dm}^{-3} \mathrm{de} \mathrm{Pe} 200 \mathrm{mg} \cdot \mathrm{dm}^{-3} \mathrm{de} \mathrm{N}$, quando se usar terra de subsolo na produção de mudas, provenientes de Latossolo Vermelho-amarelo e $300 \mathrm{mg}^{-\mathrm{dm}^{-3}}$ de $\mathrm{P}$ e $90 \mathrm{mg} \cdot \mathrm{dm}^{-3}$ de $\mathrm{N}$ quando usar Argissolo Vermelho-amarelo. Quanto à adição de $\mathrm{K}$, como as mudas se mostraram pouco exigentes, ou os teores disponíveis nos solos foram suficientes para o suprimento de suas necessidades, recomenda-se a aplicação de dose mínima testada que é de $50 \mathrm{mg} \cdot \mathrm{dm}^{-3}$ de solo, até que novos estudos com dose inferiores a esta sejam testados.

\section{GROWTH OF JACARANDA-DA-BAHIA (Dalbergia nigra((Vell.) Fr. All. ex Benth)) UNDER DIFFERENT LEVELS OF NPK}

ABSTRACT: The use of well-nourished and quality seedlings is one of the factors that contribute to the successful implementation of forest plantations. The present study aimed to verify, by means of morphological characteristics, the effects of nitrogen, phosphorus and potassium on the growth of jacarandá-da-bahia seedlings (Dalbergia nigra ((Vell.) Fr. All. in order to establish the optimal dose of each nutrient to be added in different substrates thus aiming to ensure the production of seedlings with better quality. The seedlings were produced in three types of soils: mesotrophic Ultisol (PVAm), Alic Oxisol (LVAa) and distrofic Oxisol (LVAd). The experiment was conducted under greenhouse conditions from February to June of 2006. II treatments were defined according baconian matrix, in which the nutrients (N, P and $\mathrm{K}$ ) ranged in three different doses and two additional treatments (Zero and Reference). A completely randomized design with four replications was adopted. The seedlings were planted in pots with a capacity of $2.1 \mathrm{dm}^{3}$ and 120 days after sowing the data was collected. After analyzing the data, it was concluded that for the production of jacarandá-da-bahia seedlings it is recommended an addition of dosages greater than or equal to $600 \mathrm{mg} \cdot \mathrm{dm}^{-3}$ of $P$ and 200 $\mathrm{mg} \cdot \mathrm{dm}^{-3}$ of $\mathrm{N}$ when using underground earth in the production of seedlings, from distrofic Oxisol and $300 \mathrm{mg} \cdot \mathrm{dm}^{-3}$ of $\mathrm{P}$ and $90 \mathrm{mg} \cdot \mathrm{dm}^{-3}$ of $\mathrm{N}$ when using mesotrophic Ultisol. As for the addition of $\mathrm{K}$, since the seedlings were undemanding or the available contents in the soil were sufficient to supply their needs, it is recommended the application of the minimum tested dose of $50 \mathrm{mg} \cdot \mathrm{dm}^{-3}$ of soil until further studies with lower doses is tested.

\footnotetext{
' Universidade Federal do Espírito Santo - Jerônimo Monteiro, Espírito Santo, Brasil

2 Universidade Federal de Viçosa - Viçosa, Minas Gerais, Brasil

${ }^{3}$ Universidade Federal do Espírito Santo - Alegre, Espírito Santo, Brasil
} 


\section{INTRODUÇÃO}

Dalbergia nigra, vulgarmente conhecida como jacarandá-da-bahia é uma espécie da família Fabaceaepapilionoideae de ocorrência nos estados da Bahia, Espírito Santo, Minas Gerais, Rio de Janeiro e São Paulo, na floresta pluvial atlântica (LORENZI, 2000). Na escala sucessional de uma floresta é classificada como secundária tardia, sendo raramente encontrada em regeneração natural (CARVALHO, 2003). Sua madeira, cuja densidade varia de 0,75 a $1,22 \mathrm{~g} \cdot \mathrm{dm}^{-3}$, é própria para móveis de luxo, sendo mundialmente conhecido seu emprego na construção de piano; é empregada também para acabamentos internos em construção civil, como lambris, molduras, portas, rodapés e, também, em revestimento de móveis, caixas de rádios e televisão, instrumentos musicais, etc. (LORENZI, 2000).

As utilidades da madeira dessa espécie por si só, aliado ao fato de que a espécie também é indicada para restauração de aéreas degradadas, justificam estudos que elucidem melhor os aspectos relacionados à produção de mudas visando aos plantios para fins comerciais ou ambientais.

Para se produzir mudas com qualidade há que se considerarem diversos fatores, dentre eles a fertilização adequada dos substratos, visando a garantir teores nutricionais suficientes para $\circ$ crescimento das mudas. Segundo Gonçalves et al. (2005), uma muda de boa qualidade aparenta vigor e bom estado nutricional com folhas e coloração típicas da espécie. Sendo assim, alguns estudos já foram conduzidos por diversos pesquisadores, visando a aumentar o conhecimento silvicultural do jacarandá-da-bahia. Tal como o de Marques et al. (2006a), que avaliaram o efeito da aplicação de diferentes fontes e doses de $\mathrm{N}$ no crescimento inicial dessa espécie, e observaram que mudas de melhor qualidade foram produzidas com dose de $180 \mathrm{mg} \cdot \mathrm{dm}^{-3}$ de $\mathrm{N}$, tendo como fonte o sulfato de amônio, sendo o substrato a base de Argissolo Vermelho-amarelo. Em outro estudo,
Bernardino et al. (2007), verificaram que não houve efeito significativo da elevação da saturação por bases sobre as características morfológicas e suas relações quando a saturação por bases foi igual ou superior a $14 \%$ no Latossolo Vermelho-amarelo distrófico e 4\% no Latossolo Vermelho-amarelo álico, e recomendou a utilização da calagem quando o substrato for o Argissolo Vermelhoamarelo, com elevação da saturação por bases para próximo de $60,0 \%$ e corretivo com $100 \%$ de $\mathrm{CaCO}_{3}$.

Conduziu-se este trabalho com o objetivo de verificar, por meio de características morfológicas, os efeitos do nitrogênio, fósforo e potássio no crescimento de mudas do jacarandá-da-bahia, visando a estabelecer a dose ideal de cada nutriente a ser adicionada em diferentes substratos, para garantir a produção de mudas de melhor qualidade.

\section{MATERIAL E MÉTODOS}

O experimento foi conduzido no período de fevereiro de 2006 a junho de 2006, em casa de vegetação no Horto Florestal do Instituto Federal de Educação, Ciência e Tecnologia do Sudeste de Minas - Campus Rio Pomba, em Rio Pomba, Minas Gerais. Situado nas coordenadas geográficas $21^{\circ} 14^{\prime} 31^{\prime \prime} \mathrm{S}$ e $43^{\circ}$ 09'39" O e cuja elevação é de $477 \mathrm{~m}$. O local apresentou temperatura média diária de $21,11^{\circ} \mathrm{C}$, máxima de $26,52^{\circ} \mathrm{C}$ e mínima de $17,68^{\circ} \mathrm{C}$. As médias diárias de precipitação e umidade relativa do ar foram de $7,5 \mathrm{~mm}$ e $76,66 \%$, respectivamente.

Utilizaram-se como substrato na produção das mudas, amostras de três classes de solos: Argissolo Vermelho-amarelo mesotrófico (PVAm), Latossolo Vermelho-amarelo álico (LVAa) e Latossolo Vermelhoamarelo distrófico (LVAd), predominantes na região da Zona da Mata de Minas Gerais, segundo Resende et al. (2002). Estas foram retiradas cerca de 0,30 m abaixo da camada superficial do solo, em áreas distintas próximas ao Município de Viçosa, e das quais se determinou as características físicas e químicas (Tabela I).

TABELA I Análise física e química de três tipos de solos antes da aplicação dos tratamentos para a produção de mudas de jacarandá-da-bahia.

TABLE I Physical and chemical analyses of three soil types before the application of treatments for the production of jacarandáda-bahia seedlings.

\begin{tabular}{|c|c|c|c|c|c|c|c|c|c|c|c|c|}
\hline \multirow{2}{*}{ Solo } & \multirow{2}{*}{ Classe textural } & \multirow{2}{*}{$\frac{\mathrm{pH}}{\left(\mathrm{H}_{2} \mathrm{O}\right)}$} & $\mathrm{P}$ & $\mathrm{K}$ & $\mathrm{Ca}$ & $\mathrm{Mg}$ & $\mathrm{Al}$ & $\mathrm{H}+\mathrm{Al}$ & SB & $\mathrm{T}$ & $\mathrm{V}$ & $\mathrm{m}$ \\
\hline & & & \multicolumn{2}{|c|}{$\left(\mathrm{mg} \cdot \mathrm{dm}^{-3}\right)$} & \multicolumn{6}{|c|}{$\left(\mathrm{cmol}_{\mathrm{c}} \cdot \mathrm{dm}^{-3}\right)$} & \multicolumn{2}{|c|}{$(\%)$} \\
\hline PVAm' & Franco-argilosa & 6,0 & 2,1 & 14,0 & 1,6 & 0,1 & 0,0 & 3,6 & $\mathrm{I}, 7$ & 5,4 & 32,0 & 0,0 \\
\hline $\mathrm{LVAa}^{2}$ & Argilosa & 4,8 & $\mathrm{I}, 4$ & 32,0 & 0,2 & 0,0 & 0,8 & 3,9 & 0,3 & 4,2 & 7,0 & 74,0 \\
\hline LVAd $^{3}$ & Muito argilosa & 5,2 & 0,8 & 49,0 & 0,3 & 0,0 & 0,0 & $\mathrm{I}, 8$ & 0,4 & 2,2 & 19,0 & 0,0 \\
\hline
\end{tabular}

${ }^{1}$ Argissolo Vermelho-Amarelo mestrófico, ${ }^{2}$ Latossolo Vermelho-Amarelo álico, ${ }^{3}$ Latossolo Vermelho-Amarelo distrófico 
Delimitaram-se II tratamentos, seguindo uma matriz baconiana (TURRENT, 1979), conforme é apresentado na Tabela 2, que foram dispostos no delineamento experimental inteiramente casualisado, com quatro repetições.

Os solos utilizados foram secos ao ar e passados em peneiras de malha de cinco $\mathrm{mm}$. Amostras de 2,I kg de cada solo foram pesadas em sacos plásticos de capacidade aproximada de $5 \mathrm{~kg}$. Nestas, foram adicionados e homogeneizados os sais fornecedores de cálcio e magnésio $\left(\mathrm{CaCO}_{3}\right.$ e $\left.\mathrm{MgCO}_{3}\right)$ que estavam na forma de pó, nas quantidades definidas pelos tratamentos, independente do tipo de solo.

Fez-se irrigação até a capacidade de campo do solo e esse teor de umidade foi mantido durante 20 dias com monitoramento diário por pesagens do solo mais água, para a reação dos carbonatos de cálcio e magnésio. Após esse período, a irrigação foi suspensa durante 10 dias, para facilitar a adição e homogeneização dos demais nutrientes de acordo com as quantidades definidas nos tratamentos. Para tanto, foram preparadas soluções que foram aplicadas com auxílio de pipeta graduada em $\mathrm{mL}$, contendo os nutrientes utilizando os seguintes sais: $\mathrm{NH}_{2} \mathrm{PO}_{4} \cdot \mathrm{H}_{2} \mathrm{O}$ para $\circ \mathrm{N}$ e $\mathrm{P}, \mathrm{NH}_{4} \mathrm{NO}_{3}$ para $\circ \mathrm{N}$ e $\left(\mathrm{NH}_{4}\right)_{2} \mathrm{SO}_{4}$ para $\circ$ $\mathrm{N}$ e S, $\mathrm{K}_{2} \mathrm{SO}_{4}$ para $\mathrm{S}$ e $\mathrm{K}$, e $\mathrm{KCl}$ para $\mathrm{K}$. A aplicação de solução contendo $\mathrm{N}, \mathrm{K}$ e S foram parceladas em quatro vezes $(0,30,60,90$ dias) após a semeadura.

Antes da semeadura, adicionou-se também, em todas as amostras, independente do tipo de solo, uma solução de micronutrientes, nas seguintes doses: $B=0,8$ I $\mathrm{mg} \cdot \mathrm{dm}^{-3}\left(\mathrm{H}_{3} \mathrm{BO}_{3}\right), \mathrm{Cu}=\mathrm{I}, 33 \mathrm{mg} \cdot \mathrm{dm}^{-3}\left(\mathrm{CuSO}_{4} \cdot 5 \mathrm{H}_{2} \mathrm{O}\right)$, $\mathrm{Mo}=0,15 \mathrm{mg} \cdot \mathrm{dm}^{-3}\left[(\mathrm{NH} 4)_{6} \mathrm{Mo}_{7} \mathrm{O}_{24} \cdot 4 \mathrm{H}_{2} \mathrm{O}\right], \mathrm{Mn}=3,66$ $\mathrm{mg} \cdot \mathrm{dm}^{-3}\left(\mathrm{MnCl}_{2} \cdot \mathrm{H}_{2} \mathrm{O}\right)$ e $\mathrm{Zn}=4,0 \mathrm{mg} \cdot \mathrm{dm}^{-3}\left(\mathrm{ZnSO}_{4} \cdot 7 \mathrm{H}_{2} \mathrm{O}\right)$ (ALVAREZ, 1974). Após a adição e devida homogeneização, os solos foram acondicionados nos vasos.

As sementes de jacarandá-da-bahia foram adquiridas no Setor de Silvicultura do Departamento de Engenharia Florestal da Universidade Federal de Viçosa. Cada vaso recebeu 10 sementes, efetuando-se o primeiro desbaste aos 15 dias após a emergência, deixando-se duas plantas por vaso. Após 30 dias da semeadura, um segundo desbaste foi realizado, deixando-se apenas uma planta por vaso.

Durante o período experimental, a umidade do solo foi mantida próxima de $60 \%$ da capacidade de campo, procedendo-se um monitoramento diário com o uso de balança, para esse controle, tendo como base a massa de solo e água por vaso.

A parcela experimental foi constituída por um vaso de polipropileno rígido, contendo cada um $2,1 \mathrm{dm}^{3}$ de solo e uma muda. Cada solo constituiu um ensaio distinto.

As medições de altura e diâmetro do coleto das mudas foram realizadas 120 dias após a semeadura. Em seguida, as plantas foram colhidas e subdivididas em raízes e parte aérea, lavadas em água destilada e secas em estufa a $45^{\circ} \mathrm{C}$ com circulação forçada de ar até peso constante. Depois de secas, foram pesadas em balança analítica com precisão de $0,0 \mathrm{l} g$ para determinação da massa seca da parte aérea (MSPA), das raízes (MSR) e total (MST).

TABELA 2 Descrição dos tratamentos com as devidas doses de N, P e K obtidos a partir de uma matriz baconiana, aplicados nos três tipos solos utilizados na produção das mudas de jacarandá-da-bahia.

TABLE 2 Description of the treatments with the appropriate doses of N, P and K obtained from a Baconian matrix applied in the three soil types used in the production of jacarandá-da-bahia seedlings.

\begin{tabular}{|c|c|c|c|c|c|c|}
\hline \multirow{2}{*}{ Tratamento } & \multicolumn{6}{|c|}{ Quantidade dos nutrientes aplicados } \\
\hline & $\mathrm{N}\left(\mathrm{mg} \cdot \mathrm{dm}^{-3}\right)$ & $\mathrm{P}\left(\mathrm{mg} \cdot \mathrm{dm}^{-3}\right)$ & $\mathrm{K}\left(\mathrm{mg} \cdot \mathrm{dm}^{-3}\right)$ & $\mathrm{Ca}\left(\mathrm{cmol}_{c} \cdot \mathrm{dm}^{-3}\right)$ & $\mathrm{Mg}\left(\mathrm{cmol}_{c} \cdot \mathrm{dm}^{-3}\right)$ & $\mathrm{S}\left(\mathrm{mg} \cdot \mathrm{dm}^{-3}\right)$ \\
\hline 1 & 100 & 300 & 100 & 1,0 & 0,4 & 40,0 \\
\hline 2 & 0 & 0 & 0 & 0 & 0 & 0 \\
\hline 3 & 50 & 300 & 100 & 1,0 & 0,4 & 40,0 \\
\hline 4 & 150 & 300 & 100 & 1,0 & 0,4 & 40,0 \\
\hline 5 & 200 & 300 & 100 & 1,0 & 0,4 & 40,0 \\
\hline 6 & 100 & 150 & 100 & 1,0 & 0,4 & 40,0 \\
\hline 7 & 100 & 450 & 100 & 1,0 & 0,4 & 40,0 \\
\hline 8 & 100 & 600 & 100 & 1,0 & 0,4 & 40,0 \\
\hline 9 & 100 & 300 & 50 & 1,0 & 0,4 & 40,0 \\
\hline 10 & 100 & 300 & 150 & 1,0 & 0,4 & 40,0 \\
\hline 11 & 100 & 300 & 200 & 1,0 & 0,4 & 40,0 \\
\hline
\end{tabular}


Os dados foram interpretados estatisticamente por meio de contrastes, análises de variância e regressão com o uso do programa SAEG (Sistema de Análises Estatística e Genética) (EUCLYDES, 1997). Testou-se o efeito da adição de $\mathrm{N}, \mathrm{P}$ e $\mathrm{K}$ em relação à testemunha em um contraste entre as médias do tratamento dois (sem adição de nutrientes) versus as médias dos demais tratamentos $(\mathrm{C}=\mathrm{TI}-\mathrm{IOT} 2+\mathrm{T} 3+\mathrm{T} 4+\ldots+\mathrm{TI} 0+\mathrm{TII})$.

Para a obtenção das equações utilizou-se quatro pontos, sendo três relativos às doses testadas para cada nutriente e um do tratamento de referência comum para todos os nutrientes. As curvas de respostas à adição de doses de cada nutriente resultaram de modelos de regressão nos quais os coeficientes foram testados com base nos valores do quadrado médio do resíduo da ANOVA conjunta, sendo analisada a $1 \%$, $5 \%$ e $10 \%$ de probabilidade. A partir das equações, no caso de modelos quadráticos e de raiz quadrada, foram determinadas as doses recomendadas de $\mathrm{N}, \mathrm{P}$ e K para a obtenção de $90 \%$ dos valores máximos estimados para as diversas características estudadas.

\section{RESULTADOS E DISCUSSÃO}

A adição de nitrogênio, fósforo e potássio favoreceram o crescimento das mudas, pois as médias das características avaliadas dos tratamentos que receberam a adição desses nutrientes foram superiores às do tratamento com a dose igual a zero, conforme é mostrado pelas estimativas do contraste na Tabela 3.

TABELA 3 Estimativas dos contrastes entre o tratamento sem adição de nutrientes e os demais tratamentos, testados na produção de mudas de jacarandá-dabahia.

TABLE 3 Estimates of the contrasts between the treatment without the addition of nutrients and other treatments that were tested in jacarandá-da-bahia seedlings.

\begin{tabular}{|c|c|c|c|c|c|}
\hline \multirow[b]{2}{*}{ Solo } & \multicolumn{5}{|c|}{ Estimativas do contraste } \\
\hline & $\begin{array}{c}\text { Diâmetro } \\
(\mathrm{mm})\end{array}$ & $\begin{array}{l}\text { Altura } \\
(\mathrm{cm})\end{array}$ & $\begin{array}{l}\text { MSPA } \\
(\mathrm{g})\end{array}$ & $\begin{array}{c}\text { MSR } \\
(g)\end{array}$ & $\begin{array}{l}\text { MST } \\
(\mathrm{g})\end{array}$ \\
\hline LVAd & 3,04 **** & $4 I, 4 I$ ***** & 2,38 **** & 1,37 **** & $3,77^{*} * * *$ \\
\hline PVAm & 2,49 ***** & 33,37 ***** & $1,\left.6\right|^{*} * * * *$ & $1,00 * * * *$ & $2,7 I^{\text {****** }}$ \\
\hline LVAa & $0,49 * *$ & 8,65 ***** & 0,26 **** & $0,25 * *$ & $0,50 * * *$ \\
\hline
\end{tabular}

**** significativos $(\mathrm{P}<0,0 \mathrm{I})$ ** significativos $(\mathrm{P}<0,05)$

Esse resultado era esperado, dado que os teores nutricionais originalmente existentes nos solos (Tabela I) utilizados como substratos para a produção das mudas, a exceção do nutriente K no LVAd e LVAa, são baixos conforme Alvarez et al. (1999). Isso demonstra a importância de ter nutrientes em quantidades adequadas no solo, para garantir um adequado funcionamento de todos os processos fisiológicos e metabólicos, para que a planta possa expressar seu máximo crescimento.

\section{Efeito do Nitrogênio}

Conforme é apresentado na Tabela 4, verificou-se que a adição de nitrogênio não influenciou as características diâmetro e altura, quando as mudas foram cultivadas no PVAm e LVAa. Nas mudas que cresceram no LVAd, ou não foram encontrados modelos matemáticos que se ajustassem adequadamente às respostas, ou as características não foram influenciadas de forma significativa. No entanto, a ausência de efeitos do $\mathrm{N}$ também é relatada em mudas de angico- vermelho (Anadenanthera macrocarpa (Benth.) Brenan) cultivadas em LVAd com a aplicação de $\mathrm{N}$ em diferentes doses (GONÇALVES et al., 2008).

Os efeitos de ordem quadrática observados para a MSPA, MSR e MST das mudas cultivadas no PVAm, permitem estimar as doses de $\mathrm{N}$ que proporcionará $90 \%$ de máxima produção dessas características em: $80,30 \mathrm{mg} \cdot \mathrm{dm}^{-3}$ para o MSPA (I,80 g), $87 \mathrm{mg} \cdot \mathrm{dm}^{-3}$ para o $\operatorname{MSR}(\mathrm{I}, 23 \mathrm{~g})$ e $8 \mathrm{I}, 80 \mathrm{mg} \cdot \mathrm{dm}^{-3}$ para o MST $(3 \mathrm{~g})$. Os valores encontrados nesse estudo, são próximos à dose de 72,5 $\mathrm{mg} \cdot \mathrm{dm}^{-3} \mathrm{de} \mathrm{N}$, considerada suficiente para o crescimento de mudas de gonçalo-alves (Astronium fraxinifolium), cultivadas num Latossolo Vermelho distrófico estudados por Feitosa et al. (20I I). Contudo, são menores dos que as encontradas por Marques et al. (2006a) cujos valores variaram de 120 a $196 \mathrm{mg} \cdot \mathrm{dm}^{-3} \mathrm{em}$ mudas dessa mesma espécie cultivadas em PVAm e LVAd.

Vários outros estudos com diferentes espécies florestais encontrados na literatura, mostram que os melhores resultados na produção de mudas, considerando as diferentes variáveis analisadas, ocorrem de forma geral para a aplicação de doses de $\mathrm{N}$ superiores a $170 \mathrm{mg} \cdot \mathrm{dm}^{-3}$ (CRUZ et al., 20I0; GONÇALVES et al., 2008; MARQUES et al., 2006b). Esses valores são mais próximos do verificado no presente estudo, para a MSPA nas mudas que se desenvolveram no LVAa, cujo efeito de ordem linear, indica que as maiores médias de produção de massa seca da parte aérea são alcançadas em doses superiores a $200 \mathrm{mg} \cdot \mathrm{dm}^{-3}$.

Contrário ao observado nas mudas cultivadas no LVAd, era esperado que a adição do nitrogênio ao substrato promovesse efeitos positivos no crescimento das mudas, pois a falta desse nutriente causa, dentre outros 
TABELA 4 Equações de regressão e doses estimadas de nitrogênio para as características altura, diâmetro e a massa seca da parte aérea, raiz e total em mudas de jacarandá-da-bahia 120 dias após a semeadura.

TABLE 4 Regression equations and estimated doses of nitrogen for height, diameter and dry weight of the aerial part, root and total in jacarandá-da-bahia seedlings 120 days after sowing.

\begin{tabular}{|c|c|c|c|c|}
\hline Característica & Solo & Equação & $\mathrm{R}^{2}$ & $\mathrm{DR}^{\prime}\left(\mathrm{mg} \cdot \mathrm{dm}^{-3}\right)$ \\
\hline & LVAd & $\hat{Y}=Y=1,96$ & - & 50,00 \\
\hline \multirow[t]{3}{*}{ Diâmetro (mm) } & PVAm & $\hat{Y}=Y=3,90$ & - & 50,00 \\
\hline & LVAa & - & - & - \\
\hline & LVAd & $\hat{Y}=Y=19,64$ & - & 50,00 \\
\hline \multirow[t]{3}{*}{ Altura $(\mathrm{cm})$} & PVAm & $\hat{Y}=43,03$ & - & 50,00 \\
\hline & LVAa & - & - & - \\
\hline & LVAd & $\hat{Y}=Y=0,35$ & - & 50,00 \\
\hline \multirow[t]{3}{*}{ MSPA (g) } & PVAm & $\hat{Y}=-5,61492+1,42483 * X^{0,5}-0,0666585 * X$ & 0,38 & 80,30 \\
\hline & LVAa & $\hat{Y}=0,8988+0,0084 * X$ & 0,48 & 200,00 \\
\hline & LVAd & $\hat{\mathrm{Y}}=\mathrm{Y}=0,36$ & - & 50,00 \\
\hline \multirow[t]{3}{*}{$\operatorname{MSR}(g)$} & PVAm & $\hat{Y}=-3,52882+0,876431 * X^{0,5}-0,0392698 * X$ & 0,44 & 87,00 \\
\hline & LVAa & $\hat{Y}=Y=1,25$ & - & 50,00 \\
\hline & LVAd & $\hat{Y}=Y=0,70$ & - & 50,00 \\
\hline \multirow[t]{2}{*}{ MST (g) } & PVAm & $\hat{Y}=-9,14375+2,30127 * X^{0,5}-0,105928 * X$ & 0,43 & 81,80 \\
\hline & LVAa & $\hat{Y}=Y=3,20$ & - & 50,00 \\
\hline
\end{tabular}

I DR: Dose recomendada. * significativo $(\mathrm{P}<0, \mathrm{I})$

problemas, a inibição da síntese protéica, reduzindo o processo de divisão celular e, consequentemente, o crescimento da planta (MALAVOLTA, 2006). Entretanto, nesse solo, as mudas apresentaram as maiores médias de crescimento, o que sugere que a demanda de nitrogênio pela planta, pode ter sido suprida pela fixação biológica, uma vez que a espécie é pertencente ao grupo das leguminosas, e, portanto, possui associação com bactérias fixadoras de nitrogênio.

Os efeitos observados quando as mudas foram cultivadas no PVAm podem estar relacionadas com a maior fertilidade natural desse solo que pode ter suprido as necessidade das planta. No solo LVAa, cujas médias foram as menores, verifica-se que a aplicação dos nutrientes na dose de referência pouco contribuiu para que houvesse efeitos, possivelmente pelo fato das características do solo, não terem favorecido a fixação dos nutrientes, apesar da aplicação dos nutrientes móveis ter sido parcelada, ou ainda, porque houvesse necessidade de aplicar mais nutrientes ou de melhorar as características no solo como um todo.

\section{Efeito do Fósforo}

Todas as características avaliadas responderam de alguma forma a adição do fósforo, independente dos solos em questão (Tabela 5).
Todas as características foram influenciadas linearmente quando as mudas foram cultivadas em LVAa e no LVAd, o que quer dizer, que à medida que aumenta as doses de P, ocorre aumento dos valores das características avaliadas. Dessa maneira, o maior crescimento das mudas, será alcançado em doses de $\mathrm{P}$ superiores a $600 \mathrm{mg} \cdot \mathrm{dm}^{-3}$.

Os modelos ajustados para as diversas características nas mudas cultivadas em PVAm, permitem estimar os pontos de máxima produtividade, e definir a dose recomenda de $\mathrm{P}$ para garantir $90 \%$ dessa produtividade em: $253,20 \mathrm{mg} \cdot \mathrm{dm}^{-3}$ para o diâmetro $(3,99 \mathrm{~mm}) ; 308,04 \mathrm{mg} \cdot \mathrm{dm}^{-3}$ para a altura $(50,58 \mathrm{~cm}) \mathrm{e}$ $287,06 \mathrm{mg} \cdot \mathrm{dm}^{-3}$ para a MSPA (2,06 g). Para a MSR e MST o modelo foi significativo, no entanto, não conseguiu explicar a curva de crescimento, por esta não apresentar realismo biológico, não sendo possível determinar uma dose adequada.

Os resultados desse trabalho mostram que o jacarandá-da-bahia é responsivo à aplicação de $\mathrm{P}$, o que era esperado, face aos baixos teores desse elemento encontrado originalmente dos três solos, utilizados como substrato para crescimento das mudas. Os resultados corroboram com os de outros estudos dentre os quais citam-se: Ceconi et al. (2006) em mudas de açoita cavalo (Luehea divaricata), cuja dose de P indicada é de $360 \mathrm{mg} \cdot \mathrm{dm}^{-3}$. Gonçalves et al. (2008) em mudas de 
TABELA 5 Equações de regressão e doses estimadas de fósforo para altura, diâmetro e a massa seca da parte aérea, raiz e total em mudas de jacarandá-da-bahia 120 dias após a semeadura.

TABLE 5 Regression equations and estimated doses of phosphorus for height, diameter and dry weight of the aerial part, root and total in jacarandá-da-bahia seedlings 120 days after sowing.

\begin{tabular}{|c|c|c|c|c|}
\hline Característica & Solo & Equação & $\mathrm{R}^{2}$ & $\mathrm{DR}^{\prime}\left(\mathrm{mg} \cdot \mathrm{dm}^{-3}\right)$ \\
\hline & LVAd & $\hat{\mathrm{Y}}=1,3246+0,0023^{* * * *} \mathrm{X}$ & 0,86 & 600,00 \\
\hline \multirow[t]{3}{*}{ Diâmetro $(\mathrm{mm})$} & PVAm & $\hat{Y}=-9,73945+1,46647 * * * X^{0,5}-0,0379472 * * * X$ & 0,50 & 253,20 \\
\hline & LVAa & $\hat{Y}=2,7488+0,0038^{* * *} X$ & 0,59 & 600,00 \\
\hline & LVAd & $\hat{Y}=11,442+0,0237^{* *} \times$ & 0,86 & 600,00 \\
\hline \multirow[t]{3}{*}{ Altura $(\mathrm{cm})$} & PVAm & $\hat{Y}=-28,2094+0,406592 * * * X-0,000489567 X^{2}$ & 0,99 & 308,00 \\
\hline & LVAa & $Y=25,313+0,0557 * * * x$ & 0,79 & 600,00 \\
\hline & LVAd & $\hat{Y}=0,0196+0,001 * X$ & 0,96 & 600,00 \\
\hline \multirow[t]{3}{*}{ MSPA (g) } & PVAm & $\hat{Y}=-10,9379+1,36082 * * * X^{0,5}-0,350039 * * * X$ & 0,54 & 287,00 \\
\hline & LVAa & $\hat{Y}=1,3525+0,0023 * * X$ & 0,33 & 600,00 \\
\hline & LVAd & $\hat{Y}=0,0925+0,007^{*} * X$ & 0,54 & 600,00 \\
\hline \multirow[t]{3}{*}{ MSR (g) } & PVAm & $\hat{Y}=-5,23391+0,706962 * * X^{0,5}-0,0193029 * * X$ & 0,44 & - \\
\hline & LVAa & $\hat{Y}=0,6538+0,0021 * * * X$ & 0,70 & 600,00 \\
\hline & LVAd & $\hat{Y}=0,0729+0,0018^{* *} X$ & 0,80 & 600,00 \\
\hline \multirow[t]{2}{*}{ MST (g) } & PVAm & $\hat{Y}=-16,1718+2,06778 * * * X^{0,5}-0,0543068 * * * X$ & 0,51 & - \\
\hline & LVAa & $\hat{Y}=2,0063+0,0044^{* * *} X$ & 0,49 & 600,00 \\
\hline
\end{tabular}

I DR: Dose recomendada. **** ${ }^{*} *{ }^{*}$ - significativos, respectivamente $(P<0,0 \mathrm{I}),(\mathrm{P}<0,05)$ e $(\mathrm{P}<0, \mathrm{I})$

angico-vermelho (Anadenanthera macrocarpa), definiram valores que variaram entre 150 a $250 \mathrm{mg} \cdot \mathrm{dm}^{-3}$ de $\mathrm{P}$ e Gonçalves et al. (20l0), em mudas de sabiá (Mimosa caesalpiniaefolia Benth.) encontraram valores de 312 a $600 \mathrm{mg} \cdot \mathrm{dm}^{-3}$, em ambos os casos, os valores variaram de acordo com o solo usado na produção das mudas. Cruz et al. (20I I), também encontraram valores de dose de $P$ superiores a $600 \mathrm{mg} \cdot \mathrm{dm}^{-3}$, estudando a espécie de fedegoso (Senna macranthera), cultivadas em PVAm. Todos esses resultados contrariam Carvalho (2003) que afirma que jacarandá-da-bahia não é exigente quanto a esse elemento.

\section{Efeito do Potássio}

Não foram observados efeitos significativos no crescimento das mudas de jacarandá-da-bahia, a despeito da aplicação de doses crescentes de K. As maiores médias observadas foram encontradas no LVAa e as menores médias no LVAd tal como é mostrado na Tabela 6.

Os teores de $\mathrm{K}$ existentes originalmente nos solos, é muito baixo no PVAm, baixo no LVAa, e médio no LVAd, segundo Alvarez et al. (1999). Mas podem ter sido suficientes para suprir as necessidades da mudas de jacarandá-da-bahia, sobretudo para o LVAd, o que explicaria a ausência de respostas. Silva et al. (1997),
TABELA 6 Valores médios observados e doses estimadas de potássio para altura, diâmetro e a massa seca da parte aérea, raiz e total em mudas de jacarandáda-bahia I 20 dias após a semeadura.

TABLE 6 Observed average values and estimated doses of potassium for height, diameter and dry weight of the aerial part, root and total in jacarandá-da-bahia seedlings 120 days after sowing.

\begin{tabular}{|c|c|c|c|c|}
\hline Característica & Solo & Equação & $\mathrm{R}^{2}$ & $\begin{array}{c}\mathrm{DR}^{\prime} \\
\left(\mathrm{mg} \cdot \mathrm{dm}^{-3}\right)\end{array}$ \\
\hline \multirow{3}{*}{ Diâmetro $(\mathrm{mm})$} & LVAd & $\hat{Y}=Y=2,15$ & - & 50,00 \\
\hline & PVAm & $\hat{Y}=Y=4,42$ & - & 50,00 \\
\hline & LVAa & $\hat{Y}=Y=5,14$ & - & 50,00 \\
\hline \multirow{3}{*}{ Altura (cm) } & LVAd & $\hat{Y}=Y=20,30$ & - & 50,00 \\
\hline & PVAm & $\hat{Y}=Y=47,40$ & - & 50,00 \\
\hline & LVAa & $\hat{Y}=Y=61,66$ & - & 50,00 \\
\hline \multirow{3}{*}{ MSPA (g) } & LVAd & $\hat{Y}=Y=0,30$ & - & 50,00 \\
\hline & PVAm & $\hat{Y}=Y=2,13$ & - & 50,00 \\
\hline & LVAa & $\hat{Y}=Y=3,02$ & - & 50,00 \\
\hline \multirow{3}{*}{ MSR (g) } & LVAd & $\hat{Y}=Y=0,36$ & - & 50,00 \\
\hline & PVAm & $\hat{Y}=Y=1,31$ & - & 50,00 \\
\hline & LVAa & $\hat{Y}=Y=1,66$ & - & 50,00 \\
\hline \multirow{3}{*}{ MST (g) } & LVAd & $\hat{Y}=Y=0,66$ & - & 50,00 \\
\hline & PVAm & $\hat{Y}=Y=3,44$ & - & 50,00 \\
\hline & LVAa & $\hat{Y}=Y=4,69$ & - & 50,00 \\
\hline
\end{tabular}

': Dose recomendada. 
após avaliar diversas espécies de diferentes classes sucessionais, verificou que o fornecimento de potássio na fase inicial de crescimento foi necessário para espécies pioneiras e para algumas espécies secundárias de rápido crescimento, e desnecessário para as espécies climax. Ou seja, observou que as espécies de classificação sucessional secundárias podem mesmo não responder à fertilização com o potássio, a exemplo do jacarandá-dabahia.

Resultados de ausência de efeitos quando da aplicação do K, são bem comuns na literatura, tal como foi verificado para o jacarandá-da-bahia. Outros autores como Balieiro et al. (200I) em mudas de Acacia holosericea e Acacia auriculiformis também verificaram ausência de efeitos sob aplicação de K sobre as diversas características de crescimento avaliadas. No entanto, Cruz et al. (20I0) observaram efeitos significativos a aplicação de $\mathrm{K}$ em mudas de fedegoso (Senna macranthera) indicando ser essa espécie florestal mais exigente nutricionalmente em potássio que a espécie desse estudo. Contudo, mesmo com ausência de respostas, a aplicação de $\mathrm{K}$ é importante, por ajudar no crescimento da planta como resultado do equilíbrio entre $\mathrm{N}, \mathrm{P}$ e K , além de reprimir a absorção excessiva do magnésio (MARSCHNER, 1995).

\section{CONCLUSÕES}

Na produção de mudas de jacarandá-da-bahia, recomenda-se a adição doses iguais ou superiores a 600 $\mathrm{mg} \cdot \mathrm{dm}^{-3}$ de $\mathrm{Pe} 200 \mathrm{mg} \cdot \mathrm{dm}^{-3}$ de $\mathrm{N}$, quando se usar terra de subsolo na produção de mudas, provenientes de Latossolo Vermelho-amarelo e $300 \mathrm{mg} \cdot \mathrm{dm}^{-3}$ de $\mathrm{P}$ e 90 $\mathrm{mg} \cdot \mathrm{dm}^{-3} \mathrm{de} \mathrm{N}$ quando usar Argissolo Vermelho-amarelo. Quanto à adição de $\mathrm{K}$, como as mudas se mostraram pouco exigentes, recomenda-se a aplicação de dose mínima testada ( $50 \mathrm{mg} \cdot \mathrm{dm}^{-3}$ de solo), até que novos estudos com doses inferiores a esta sejam testados.

\section{REFERÊNCIAS}

ALVAREZ, V. H. Equilíbrio de formas disponíveis de fósforo e enxofre em dois Latossolos de Minas Gerais. 1974. 125 f. Dissertação (Mestrado em Solos e Nutrição de Plantas) - Universidade Federal de Viçosa, Viçosa, 1974.

ALVAREZ, V. H.; NOVAIS, R. F.; BARROS, N. F.; CANTARUTTI, R. B.; LOPES, A. S. Interpretação dos resultados das análises de solo. In: RIBEIRO, A. C.; GUIMARAES, P. T. G.; ALVAREZ, V. H. (Ed.). Recomendação para o uso de corretivos e fertilizantes em Minas Gerais: 5. aproximação. Viçosa, MG: Comissão de Fertilidade do Solo do Estado de Minas Gerais, 1999. p. 25-32.
BALIERO, F. C.; OLIVEIRA, I. G.; DIAS, L. E. Formação de mudas de Acacia holosericea e Acacia auriculiformis: resposta a calagem, fósforo, potássio e enxofre. Revista Árvore, Viçosa, v. 25, n. 2, p. 183-191, 2001.

BERNARDINO, D. C. S.; PAIVA, H. N.; NEVES, J. C. L.; GOMES, J. M.; MARQUES, V. B. Influência da saturação por bases e da relação $\mathrm{Ca}: \mathrm{Mg}$ do substrato sobre o crescimento inicial de jacarandá-da-bahia (Dalbergia nigra (Vell.) Fr. All. ex Benth.). Revista Árvore, Viçosa, v. 3I, n. 4, p. 567-573, 2007.

CARVALHO, P. E. R. Espécies arbóreas brasileiras. Brasília: EMBRAPA Informação Tecnológica; Colombo: EMBRAPA Florestas, 2003. 1039 p.

CECONI, D. E.; POLETTO, I.; BRUN, E. J.; LOVATO, T. Crescimento de mudas de açoita-cavalo (luehea divaricata mart.) sob influência da adubação fosfatada. Cerne, Lavras, v. I2, n. 3, p. 292-299, 2006.

CRUZ, C. A. F.; PAIVA, H. N.; CUNHA, A. C. M. C. M.; NEVES, J. C. L. Crescimento e qualidade de mudas de fedegoso cultivadas em Latossolo Vermelho-Amarelo em resposta a macronutrientes. Scientia Forestalis, Piracicaba, v. 39, p. 2I-33, 20 II.

CRUZ, C. A. F.; PAIVA, H. N.; NEVES, J. C. L.; CUNHA, A. C. M. C. M. Resposta de mudas de Senna macranthera (Dc. Ex Collad.) H.S. Irwin \& Barnaby (Fedegoso) cultivadas em Latossolo Vermelho-amarelo distrófico a macronutrientes. Revista Árvore, Viçosa, v. 34, n. I, p. I3-24, 2010.

EUCLYDES, R. F. Manual de utilização do programa SAEG (Sistema para Análises Estatísticas e Genéticas). Viçosa, MG: UFV, 1997. 59 p.

FEITOSA, D. G.; MALTONI, K. L.; CASSIOLATO, A. M. R.; PAIANO, M. O. Crescimento de mudas de gonçalo-alves (Astronium fraxinifolium) sob diferentes fontes e doses de nitrogênio. Revista Árvore, Viçosa, v. 35, n. 3, p. 40 I-4II, 2011 .

GONÇALVES, E. O.; PAIVA, H. N. de; NEVES, J. C. L.; GOMES, J. M. Crescimento de mudas de angico-vermelho (Anadenanthera macrocarpa (Benth.) Brenan) sob diferentes doses de macronutrientes. Revista Árvore, Viçosa, v. 32, n. 6, p. 1029-1040, 2008.

GONÇALVES, E. O.; PAIVA, H. N. de; NEVES, J. C. L.; GOMES, J. M. Crescimento de mudas de sansão-do-campo (Mimosa caesalpiniaefolia Benth.) sob diferentes doses de macronutrientes. Scientia Forestalis, Piracicaba, v. 38, n. 88, p. 599-609, 2010.

GONÇALVES, J. L. M.; SANTARELLI, E. G.; MORAES NETO, S. P.; MANARA, M. P. Produção de mudas de espécies nativas: substrato, nutrição, sombreamento e fertilização. In: GONÇALVES, J. L. M.; BENEDETTI, V. (Org.). Nutrição e fertilização florestal. Piracicaba: Instituto de Pesquisas e Estudos Florestais, 2005. cap. II. 
LORENZI, H. Árvores brasileiras. Nova Odessa: Plantarum, 2000. v. I, 352 p.

MALAVOLTA, E. Manual de nutrição mineral de plantas. São Paulo: CERES, 2006. 638 p.

MARQUES, V. B.; PAIVA, H. N. de; GOMES, J. M.; NEVES, J. C. L.; BERNARDINO, D. C. de S. Efeito de fontes e doses de nitrogênio sobre o crescimento inicial e qualidade de mudas de jacarandá-da-bahia (Dalbergia nigra (Vell.) Fr. All. ex Benth.). Revista Árvore, Viçosa, v. 30, n. 5, p. 725-735, $2006 a$.

MARQUES, V. B.; PAIVA, H. N. de; GOMES, J. M.; NEVES, J. C. L.; BERNARDINO, D. C. de S. Efeitos de fontes e doses de nitrogênio no crescimento de mudas de sabiá (Mimosa caesalpiniaefolia Benth.). Scientia Forestalis, Piracicaba, n. 7I, p. 77-85, 2006b.
MARSCHNER, $H$. Mineral nutrition of higher plants. London: Academic, 1995. 889 p.

RESENDE, M.; CURI, N.; REZENDE, S. B.; CORRÊA, G. F. Pedologia: base para distinções de ambientes. 4. ed. Viçosa, MG: Neput, 2002. 338 p.

SILVA, I. R.; FURTINI NETO, A. E.; CURI, N.; VALE, F. R. do. Crescimento inicial de quatorze espécies florestais nativas em resposta à adubação potássica. Pesquisa Agropecuária Brasileira, Brasília, v. 32, n. 2, p. 205-2।2, fev. 1997.

TURRENT, F. A. Uso de uma matriz mixta para la optimización de cinco a ocho factores controlables de la producción. Chapingo: Rama de Suelos, Colégio de Postgraduados, 1979. 65 p. (Boletim Técnico, 6). 